Voix et Images

voixetimages

\title{
La fondation fantastique
}

\section{Philippe Haeck}

Volume 8, numéro 3, printemps 1983

Jacques Ferron

URI : https://id.erudit.org/iderudit/200399ar

DOI : https://doi.org/10.7202/200399ar

Aller au sommaire du numéro

\section{Éditeur(s)}

Université du Québec à Montréal

\section{ISSN}

0318-9201 (imprimé)

1705-933X (numérique)

Découvrir la revue

\section{Citer cet article}

Haeck, P. (1983). La fondation fantastique. Voix et Images, 8(3), 427-435.

https://doi.org/10.7202/200399ar d'utilisation que vous pouvez consulter en ligne.

https://apropos.erudit.org/fr/usagers/politique-dutilisation/ 


\title{
La fondation fantastique
}

\author{
par Philippe Heeck. Collège de Maisonneuve
}

\section{1 - LE RetOUR AU PAYS NATAL}

Au commencement il y a le Ciel de Québec, notre grand et seul roman d'apprentissage. Grande chronique d'événements rels, symboliques et imaginaires à avoir lieu ici en 1937. Cette anné là un jeune poète publiait Regards et Jeux dans f'espace et un jeune professeur de dessin, Paul-Emile Borduas, se voyait consacrer un premier article dans la Revue moderne. Maurice Duplessis, un peu moins jeune, chef de l'Union Nationale, était premier ministre.

J'ai lu à vingt-huit ans le Ciel de Québec: c'était en décembre 1974. "J'ai lu ce livre avec plaisir." C'est tout ce que je note à la fin de ma première lecture. Cette grande chronique était parue à l'automne 1969. A un lancement de livres de l'Aurore à l'Hôtel Iroquois, j'avais parlé un peu à Lévy qui, je ne sais pourquoi, commença à m'entretenir du Ciel de Québec qu'il considérait comme le livre ayant approché le plus du style épique ou plutôt de l'épopée dont il rêvait. Mon meilieur professeur de littéra ture québécoise ancienne aura été Lévy; pour le versant moderne çaura été Patrick Straram le Bison ravi. Deux hommes passionnés par les livres comme Jacques Ferron; mais le seul qui sourit est le troisieme.

Quand on nait dans un non-pays il arrive souvent qu'on ne sache rien de la litterature parce que l'élite communique avec les chefs-d'oeuvre des grands pays et que la masse s'annule dans les non-communications de masse. On a des professeurs d'université qui trouvent la littérature du non-pays bien pauvre, on a des secrétaires qui lisent les gros best-sellers qui viennent d'être produits à grands renforts publicitaires.

Je suis arrivé à Ferron par Lévy, je suis arrivé à Lévy parce que j'avais à rendre compte de Don Quichotte de la Démanche. Le Ciel de Québec, Don Quichotte de Ia Démanche: deux livres qui me sont essentiels pour comprendre où je vis. Deux livresboucles. Deux livres en trente-trois parties. Le Ciel de Québec a trente-quatre chapitres et une conclusion mais les chapitres XXIV et XXV sont de trop: Ferron voulait les supprimer dans l'édition parue chez VLB éditeur mais Lévy l'a convaincu de n'en rien faire. D'un point de vue ancien, celui de l'économie du récit, Lévy a tort, d'un point de vue moderne, la naissance des différences, il a raison: il fallait, peu importe comment, que Ferron fasse croiser dans son livre, qui est devenu le nótre. Saint-Denys 
Garneau et Borduas. Pourquoi? Je le dirai un peu plus loin: Ferron conversant avec Garneau et Borduas c'est tout l'apprentissage québécois qui se trame, ses trois voies.

Si je n'avais pas écrit je ne serais peut-être jamais revenu au Québec. On sait que les intellectuels d'ici ont toujours eu beaucoup de facilité à s'exiler (de l'extérieur, de l'intérieur, peu importe). Écrire engage quelqu'un à tâter, sentir la langue qui passe dans sa bouche. Étudier dans un département de lettres la plupart du temps éloigne de soi, écrire ramène à soi. On recycle souvent les enseignants, les enseignantes de l'élémentaire et du secondaire: il est dommage qu'on oublie ceux du niveau universitaire (qu'on lise le bon texte critique du collectif étudiant, "Plaisir et institution", écrit pour le colloque sur "La nouvelle écriture" en février 1980 à l'Université du Québec a Montréal'). Tout est à faire ici: a) arriver à s'aimer assez pour considérer les écritures produites ici comme les plus importantes pour nous; bl enseigner ces écritures sans les écraser sous des grilles étrangères soit parce qu'elles sont nées à l'intérieur d'autres cultures pour répondre à des histoires-géographies différentes, soit parce qu'elles méconnaissent la spécificité de la scientificité littéraire qui ne saurait être celle des sciences de la nature où les lois générales, les mesures mathématiques dominent lle geste d'écrire est encore peu connu: les étudiants n'ont pas tort de parler du "complexe de crispation" des professeurs, de contester un enseignement qui fait d'eux des juges sans pratique d'écriture); cl foncer résolument vers l'écriture, ce n'est qu'en écrivant qu'on arrive à rire de soi et à devenir soi.

Pour parler de Ferron, me répéter continuellement la proposition d'Anne Hébert dans "Poésie, solitude rompue" (1960): "Toute facilité est un piège". Ferron marche droit en faisant beaucoup de détours: par là il s'éloigne de la norme qui consiste à marcher tout croche en suivant beaucoup de droites.

Je suis venu à Ferron après le plaisir pris aux torsions mallarméennes, l'étude forcée des hâtes structuralistes à cadastrer le langage. Mon Ferron n'est pas celui des Contes: je ne les ai pas encore lus. C'est celui des récits: les Confitures de coings, le Ciel de Québec, l'Amélanchier (ces récits, le début d'une bible québécoisel. Ferron a rejoint dans mon ciel les raisons de vivre heureux de Ponge, les débordements de légèreté de Cixous. Ponge, Cixous, Ferron, ma grande triade. Mais si j'aime Ponge et Cixous, j'aime encore plus Ferron pour la raison simple-complexe que sa langue est ma langue natale.

"Monseigneur Camille, de la lignée humaniste des prélats québecquois, homme bon, discret et de bonne compagnie, disait sa messe au Précieux-Sang, dans la basseville. Chaque matin, (...)". C'est le début du chapitre un. Voila la phrase de Ferron. Elle a le tour de tout rassembler dans sa main: l'articulation nette de la prose du dix-huitième siècle français qui a tant eu de goût pour l'histoire (Montesquieu et les Encyclopédistes), la gentillesse de Giraudoux (lit-on encore cet écrivain qui sentait, voyait, entendait tout pour la première fois, qui avait une envie extrême du matin où tout apparaît neuf, lavé des poussières de la nuit), le goût des réalités cultureiles - ici la religion et l'humanisme - qui structurent fortement, à leur insu, les individus et les collectivités, un style entre l'écriture et la parole, une posture qui mêle le styliste et le conteur (d'où ce vaet-vient constant dans son écriture entre le détachement et le rapprochement, la politesse du style et le clin d'oeil complice, la critique et la bonté). Voila comme j'aime décrire la phrase d'un individu: cela n'a rien à voir avec la description structura 
liste, rhétorique, sémiotique, quand les structures, les figures, les signes sont figés en quelque système exsangue. $\dot{A}$ un universitaire qui critiquait l'écriture d'avant-garde pour son goût de l'intertextualité, du texte se disant texte, qui lui-même produisait un discours où les figures étaient nommées pour elles-mêmes, qui voulait savoir mon avis sur sa communication, je n'ai pu que répondre que je me situais ailleurs, qu'il me semblait évident que tout discours était fait de discours, que si on voulait sortir de l'impasse il fallait en arriver à considérer l'écriture comme la prise de la parole par un.individu qui se sert de l'écriture pour avancer dans un questionnement, énoncer des valeurs. II n'y a d'écriture qu'engagée dens une parole. Pour le dire plus vertement: il n'y a que la parole qui déniaise.

Le retour au pays natal est le retour à la langue maternelle. Le métis Henry Sicotte à qui on demande d'où il tient ce qu'il sait répond: "Je l'avais appris de ma mère au visage long, de ma mère triste et intelligente, un grand fichu sur les épaules, la seule femme que j'ai aimée, qui me parle encore à l'oreille et qui ne cesse de m'instruire". Ne pas confondre la langue maternelle avec le discours scientiste: "Surtout, on (il s'agit des auteurs de la Logique de Port-Royah oubliait que ces mots, appris durant la première enfance, sont bien plus que des signes rationnels et qu'avant de parler en termes on parle pour parler, par plaisir; que la vitalité d'une langue réside justement dans ce qui l'empêche d'être un instrument scientifique", ('La part du grimoire" dans Du fond de mon arrière-cuisine). Contre la réduction des langages artificiellement univoques il $y$ aura toujours, a moins qu'on assassine écrivants et parleurs, écrivantes et parleuses, la complexité émouvante de la langue maternelle - c'est de cette émotion que lẹs analystes tirent leurs profits: ils font payer chèremenț ce qui a été donné et qu'on a oublié, ou ce qui n'a pas été donné parce que l'espèce humaine, à se presser tant pour sa survie, oublie le plaisir de nommer, de parler.

En pays colonisé, c'est-d-dire en non-pays, il y a toujours des intellectuels internationalistes qui se hâtent d'oublier la langue qu'ils ont apprise dans les bras de leur mère, sur les genoux de leur père, dans la rumeur du voisinage. Qu'on se le mette dans la tête: on ne fera jamais un bon livre québécois si on n'entend pas la musique, la beauté, la puissance (mettons-en c'est pas de l'onguentl de nos parlures, de nos ecritures. II y en a qui pensent faire des livres la langue coupée: il se trouve toujours des eunuques pour applaudir.

\section{3 - UN GRAND ÉCRIVAIN CATHOLIQUE}

En janvier 1981: "Voilà la deuxième fois que je traverse cette chronique: je pense toujours plus que Ferron a écrit la plus grosse partie d'une bible en québécois". J'ai dit après d'autres qu'une bonne preuve de la fadeur, de l'échec de l'enseignement contrôlé par l'Église catholique québécoise jusqu'à 1960 était l'absence de grands écrivains catholiques. Où étaient nos Léon Bloy, Charles Péguy, Paul Claudel, Charles Du Bos, Georges Bernanos, Jacques Maritain? Voilà le type de question qui ravalait notre littérature au niveau de la médiocrité. D'ailleurs comment aurait-on pu y répondre puisqu'on ne la lisait pas. Ce n'est qu'une fois qu'on a dépassé un anticléricalisme facile qu'il devient possible de lire des textes écrits par des catholiques; il n'y a pas de raison pour que tous leurs textes soient dénués de valeur critique - je tiens /'Am- 
poule d'or de Léo-Paul Desrosiers ou tel article du frère Marie-Victorin comme des textes qui font bouger nos représentations.

En lisant le Ciel de Québec, en ruminant cette grande chronique, je me suis rappelé la foi de ma jeunesse sans la considérer comme contraire à la modernité, à vingt-cinq ans $j$ 'ai abandonné l'Église à cause du sentiment qu'elle renforçait l'aliéna tion de l'individu au lieu de travailler à sa libération, je me suis souvenu qu'il y a eu dans ma vie un Monseigneur Camille qui était un abbé lettré: son défaut n'était pas dans le domaine religieux mais dans le domaine littéraire: nourri par les oeuvres françaises, son maître était Valéry, il dédaignait les oeuvres d'ici. Un abbé lettré qui réussissait à vivre dans un équilibre fragile entre la foi et la raison, un abbé qui s'est toujours moqué comme Ferron des Monseigneur Cyrille qui voient l'enfer partout, qui a toujours préféré un petit péché à un grand malheur ('Seigneur, ne vous en déplaise, il me parait plus simple de l'imaginer saoul que mort. Entre un petit péché et un grand malheur, je choisis le petit péché.").

Je ne l'ai pas su tout de suite, tant d'idées reçues allaient à l'encontre, la pré tention même de l'auteur à être athée, puis un bon matin j'en ai été sûr: Jacques Ferron est notre grand écrivain catholique. Décidément au Québec nous sommes bien originaux et détraqués, les contradictions partent à rire dans une même limonade (un même limon, si vous préférez). Ce qu'il y a de beau dans le Ciel de Québec c'est de devenir catholique alors qu'il ne reste dans les églises que des vieux, des femmes et des enfants. Quand l'église est forte Ferron décroche Dieu du ciel, quand l'Église est écroulée Ferron remet Dieu dans le ciel - voilà l'écrivain que j'aime, celui qui se fait l'avocat du diable, qui en toutes circonstances, rappelle à ses concitoyens qu'ils(elles) pourraient agir autrement.

A lire la bonté dans le Ciel de Québec j'ai envie de renouer avec l'enseignement évangélique parce que cette bonté je la mets moins au crédit de l'individu Ferron qu'à celui de l'Église qui, malgré elle souvent, enseigne la charité et l'espérance, vertus auxquelles j'ai peut-être toujours eu foi. Me plaît ce Mgr Turquetil, "grand personnage dans la mythologie des Oblats de Marie-Immaculée" qui se déclare la "grosse vache" d'un enfant qui lui demande s'il fait la vache, qui ne craint pas de frôler l'hérésie pour mieux aimer: "Moïse Chrétien, je suis ému; votre éloquence m'a touché. Si vous dites ce que pensent vos nations et votre peuple, en vérité, en vérité, je ne connais pas de village plus catholique que le vôtre, car tout a commencé par le Verbe et le Verbe s'est fait chair pour prendre bouche et mieux s'exprimer..." Me plait que Ferron dès l'ouverture de son livre fasse jaser Dieu avec Mgr Camille; j'aime qu'on fasse jaser Dieu: c'est le mettre à bonne hauteur, à hauteur de saveur d'homme, il est bon que Dieu salue son créateur (je préfère l'amour réciproque - Dieu jasait bien aussi dans les livres de Péguy, je pense encore à ce Dieu paysan qui est une bien belle invention parce qu'elle mêle bonté et rudesse, ce qui m'émeut toujours).

Les écritures fortes naissent souvent de s'inscrire contre une institution puissante qui veut fixer les identités. Le commerce de Dieu aura été ici une entreprise considérable: Ferron en témoigne dans le Ciel de Québec. Comprendre que Garneau et Ferron en étant à côté de l'Église, en ayant des rapports difficiles avec Dieu, ont peutêtre paradoxalement donné une meilleure idée de Dieu et de l'Église aux faux mécréants. 
Garneau et Ferron, François d'Assise et Rabelais, Bernanos et Claudel, la déchirure et la couture.

J'aimerais être un catholique communiste qui trouve, Jésus correct mais n'a pas d'entretien avec Dieu.

\section{3 - UN HOMME DES LUMIERES}

Sa mère l'avait appelé Jean-Jacques parce qu'elle aimait Rousseau, lui il n'a pas détesté écrire des contes comme le faisaient Voltaire et Diderot.

Un homme des lumières ça n'aime pas l'imbécillité: quel plaisir que la volée que fait manger au Jésuite Papin, anti-communiste, I'honorable Chubby. Quel plaisir que l'hystérie de Mgr Cyrille. Quel plaisir que d'entendre l'écart entre le paraitre et l'être, que de voir nos vanités, nos roueries, défaites et aimées.

Saint-Denys Garneau, Paul-Ėmile Borduas, Camille Roy: tous les trois sur la table d'ecriture de Ferron. Le jeune poète blessé, le courageux maître à penser, l'amateur attentif de nos lettres. Qu'est-ce qu'il en fait? II les accomplit en faisant exister le pays qui aurait tué le premier, exilé le deuxième, enchanté le troisième. Ferron nous lave de nos blessures, nous fait revenir de nos exils, nous secoue de nos enchantements faciles. Quand on parle des écritures québécoises on est vite ramené à trois grands courants: a) la ligne asilaire, la réserve des fragiles; b) la ligne politique, la réserve des nationalistes; cl la ligne gentille, la réserve des régionalistes, des pasticheurs. Avec Ferron tout ça est dépassé. L'oeuvre de Ferron appartient à une quatrième ligne, ceile de la modernité, la ligne critique. C'est cette ligne que j'aime le plus, celle qui a été le moins enseignée - Ferron note que la revue dynamique des années trente était celle d'Albert Pelletier, les Idées, qu'on a complètement oubliée au profit de la Reléve. revue conservatrice de moindre qualité.

Pourquoi la critique prend-elle si difficilement au Quebec? Faiblesse de colonisés culturels bons à répéter les idées d'ailleurs, bonté niaise de catholiques qui ne veulent pas chasser les vendeurs du temple, absence de maitres à penser ou de femmes de connaissance (Ferron note que Borduas a eu un maître, Garneau des amis).

"- Monsieur Borduas, est-il possible de créer? - Ma foi, je n'en sais rien. Ce n'est pas en posant de telles questions qu'on fera de bons tableaux. II vaut mieux résourdre l'énigme en peignant tout simplẹment." Jacques Ferron ne m'apprend pas autre chose: la création arrive en parlant, en écrivant. Une universitaire me disait qu'elle ne pourrait écrire tant qu'elle n'aurait pas la maîtrise de la langue. Elle avait un doctorat es lettres et ne savait pas ce qui est au commencement: qu'on ecrit pour se maîtriser, et que lorsqu'on y est arrivé on continue à écrire pour oublier la maîtrise, pour ne pas cesser de connaître cette joie qu'il y a à avancer sans être sûr de ses pas, joie de la découverte, de l'apprentissage.

Si Ferron accomplit Garneau, Borduas, Roy, c'est qu'ils ont longtemps mariné dans sa bouche. Et un beau jour qu'il était malade plus que de raison, il a décidé de les avaler en les écrivant; alors il a bien ri et la santé lui est revenue. 
Ferron est plus qu'un homme des lumières, il est plus fort de ne pas croire comme ils ont cru au triomphe inéluctable de la raison. Sa puissance vient du brin de folie québécoise qui tient ensemble la vieille raison révolutionnaire européenne et l'encore plus vieille charité chrétienne enseignée par un jeune juif. Et puis je vais vous dire la vérité: Ferron n'est pas un homme des lumières, il le dit pour le faire croire, pour qu'on comprenne sa syntaxe délurée, mais il est ailleurs: dans le froc de Rabelais où il prie et chie, s'enthousiasme pour le chanvre indien ou les voyages des explorateurs aux pôles. Un homme de la Renaissance, oui.

"il fallut désormais créer, inventer son monde à soi, peindre ce qu'on n'avait jamais vu et qu'on apercevait pour la première fois en peignant. Étions-nous des dieux pour pouvoir oeuvrer ainsi? Ou étions-nous des fous?"

"On recourt à moi, l'inquiétant, pour se rassurer. Bah! II en a toujours été ainsi."

"Quand on veut faire la révolution, pensez-vous qu'on s'annonce?"

Exercice de philologie. "De leurs tournées en province, les politiciens retiennent au moins deux choses, la première qu'à pisser sur la chaussée on s'éclabousse, la deuxième que pour ne pas s'éclabousser, il ne faut tout de mêrne pas trop s'avancer et tomber dans le fossé." Cette phrase relève-t-elle de l'humour du siècle des Lumières ou du rire de la Renaissance?

Là où on ne rit pas il ne peut y avoir ni connaissance ni naissance. À qui apprécie l'humour je demanderais d'examiner l'hypothèse suivante: l'humour est né de la tristesse des sympathisants de la Révolution à la suite des faillites de cette dernière (il serait donc né en Angleterre et en France à la fin du dix-huitième siècle), le rire est né de la joie des hérétiques à tenter d'agir sans cesse de meilleures utopies (il secouerait tout le Moyen Âge européen et s'accomplirait dans le gosier de Rabelais). Le sourire de l'humour est inframince: il risque, sans le savoir, de servir la cruaute; it fait rire mais ne change rien, il s'accommode de la tristesse. Le rire lui n'aime pas la tristesse de l'humour, il préfère la santé de la critique qui ne s'accommode que de la joie de vivre. L'humour est plein de feintes, il a peur des tyrans; le rire rit de toutes ses dents et il appelle un tyran un tyran. Ferron dit: "les universités (...) ne sont que des lieux d'internement hautement perfectionnés".

Ferron aime les symboles; l'homme des lumières en dégonfle plusieurs (Garneau par exemple), le catholique en fabrique d'autres (le village des Chiquettesł. II aime les symboles qui, respectant les différences biographiques, historiques, géographiques, rassemblent les individus; et critique sans ménagement les symboles qui, prétendant rassembler les citoyens, les séparent et les maintiennent sous la tyrannie.

\section{4 - L'AVENTURe, Le VOYAge, la bOUCle}

Pourquoi je lis /e Ciel de Québec aujourd'hui. Pourquoi je lis Ferron. Une envie forte de m'enquébécoiser, de me laisser caresser par ma lange maternelle. Pour enquébécoiser Frank-Anacharcis, le fils du bishop Scot, Ferron ne trouve pas mieux à la fin du livre que de lui faire passer une nuit d'amour avec la putain Georgette au "sein étonnant avec un grand cerne rose". Ce rose m'en rappelle un autre, celui dont parle Borduas dans Projections libérantes: "J'imagine volontiers que, si Renoir eut vécu cent 
ans et plus, ses dernières toiles eurent été peintes d'un seul pétale de rose fait d'une infinie variété de tons, d'un clitoris remplissant le tableau d'une multitude de petites touches de chair rose et bleue."

L'aventure commence ici, voilà ce qui est donné à lire dans le Ciẹ/ de Québec. il n'y a de vrai roman que d'aventure, de voyage: on part d'un point pour revenir au même point, changé. Cela est vrai de personnages du roman (Frank-Anacharsis et Eurydice, le métis Sicotte et la capitainesse Eulalie, Orphée et l'abbé Bessette l'incendiaire) mais cela est aussi vrai de moi lecteur. Je pars de Montréal avec une culture parisienne inoculée à l'université et je reviens à Montréal avec une culture québécoise semée dans les patates de mon grand-père. Ferron m'a montré son manche à balai ét j'ai vu qu'il était bon et dur, que c'était le sourcier qui me convenait puisqu'il aimait comme moi: la curiosité, le vert et les grands soleils, Jeanne d'Arc et la Chartreuse de Parme, les tisserands de Madeleine Parent et les livres Ion trouve ces indices dans ses réponses au questionnaire Marcel Proust publiées dans Quand les écrivains québécois jouent le jeu h. Je suis parti indifférent à Dieu et à l'Église ou le prétendant, et je suis revenu avec l'envie qu'il y ait quelque chose qui les remplace. Je suis parti seul et je suis revenu en sentant en moi plein de complices: "L'air de rien, cela peut devenir l'amitié secrète de tout un peuple pour lui-même" (Du fond de mon arrière-cuisine).

Lire le Ciel de Québec aide à "voir le nouveau de l'ancien, et non seulement l'ancien dans le nouveau"2 ce qui fait s'écrouler la logique de l'identité fixe, ou la conception de l'histoire comme série de ruptures. Le Ciel de Québec est un livre aussi moderne que Don Quichotte de la Démanche ou que I'Amer ou le Chapitre effrité. Le nouveau dans le livre de Ferron: la multiplicité des histoires, la complexité du réel, le hasard, la joie langagière, le déplacement des identités, la continuité de l'histoire. Mon dou que ça fait du bien de se sentir histoire et changement: ce qui s'appelle être et devenir.

Lire Ferron: une joie de l'esprit qui fait que mes orteils sentent mieux la terre. Ferron permet de penser l'univers lié, liant: quel repos que d'étre au milieu du monde plutôt qu'à son avant-garde. Je me bats pour être au milieu du monde, les pieds dans la terre québécoise, l'intelligence dans les paroles d'ici. J'ai trop longtemps été séparé de moi.

La complexité de la grande chronique de 1937 tient, bien sûr, d'abord à l'écriture extrêmement mobile de Ferron mais également aux rapports entre plusieurs couches mythologiques: mythes grecs (Orphée et Eurydice, Calliope et Apollon), chrétiens, (Dieu et Lucifer, le Christ et l'Antéchrist, les Rois mages et l'enfant rédempteur, les miracles, sainte Eulalie, etc.), amérindiens (le voyage dans le Grand Nord pour ramener les âmes mortes, l'étalon Étoile Noire), canadiens (Louis Riel, Mgr Turquetil, la police montée, le missionnaire, etc.), québécois (Garneau, Borduas, Roy, les coureurs de bois et les putains, etc.), ferronniens (le village des Chiquettes, l'espace souterrain, le simple d'esprit, le médecin, l'incendiaire, la capitainesse, etc.l. Tout cet enchevêtrement de mythes rious donne un portrait de nous-mêmes, un ensemble de traits qui ne nous oriente plus simplement du cóté du tragique comme il arrivait trop souvent, mais tout autant du côté d'une joie gaillarde. Ce que Ferron nous apprend ce n'est pas le Québécois pure laine, mais la bonté du métissage, la complexité de l'histoire dans une géographie. 
"Je vais partir je ne sais où, à l'aventure. Je suivrai la piste des bisons." Cette piste ramène le métis Henry Sicotte au pays de son père, le Québec.

\section{5 - UN PAYS ENCORE VERT}

Transports. Sur les chevaux, il y a un Mandan, le dernier de sa tribu, ou Eurydice, la fille de Cotnoir, qui va à sa mort parce qu'Orphée est trop frileux. Les chiens sont grands, il faut les promener, l'abbé incendiaire et le monseigneur hystérique en ont peur - chiens de l'enfer. Les automobiles transportent des politiciens ou des monseigneurs qui vont chanter la pomme aux pauvres gens, ou encore Cotnoir vers sa mort. Quant aux trains, on $y$ transporte des chevaux sauvages ou on y fait une déclaration d'amour.

L'ancien et le nouveau, les chevaux et les autos, les chiens et les trains. Tout ça du côté de la puissance. La limousine cardinalice et les grands chiens du bishop (lui-même une grande échelle, un grand fanal). Les chevaux des prairies et le rapide Montréal-Québec. Tout ça pour dire que s'il y a du bon Dieu dans le Cie/ de Québec, il y a aussi du diable qui rit sous cape, du diable dont les yeux brillent dans les dents des chiens. Ce qui est surprenant chez Ferron vient d'être en même temps un chien qui mord et une mère qui embrasse, un paysan qui jase avec Dieu et un abbé qui lit Voltaire.

A regarder tout ce monde qui circule dans le Ciel de Québec j'en viens à la constatation que la vie est du côté des hommes d'Église (Mgr Camille, le bishop Scot. le cardinal Turquetil, le curé Rondeau, l'abbé Surprenant) et des femmes d'Etat (Calliope, l'intellectuelle communiste, Eulalie, la femme sage de sa tribu, Mme Casgrain, la militante socialistel. Les hommes d'État et les femmes d'Église sont plutôt du côté de la morte grise.

Je pense parfois à Eurydice et j'ai peur d'être Orphée - j'aimerais mieux mettre mes pas dans ceux de François-Anacharcis. Les hommes d'église ont des servantes; les hommes politiques paient des putains, les hommes cultivés sont trop tristes . pour retenir l'amour qui passe à côté d'eux, les hommes d'aventure aiment les chevaux et rêvent à leur mère, les pères boivent l'admiration de leurs filles. Je vous laisse deviner ce qui manque.

Au Québec tout arrive-t-il en retard? Au moment où de bons livres commencent à se fabriquer ici, qui nous seraient oeuvres de fondation, voilà qu'on claironne que l'avenir est à l'audio-visuel, à l'ordinateur-crétin, à la créativité-gadget. Vit-on plus allègrement dans une maison sans fondation. Québécois caméléons, zouaves de l'empire technologique. Qui dira que j'exagère, que je suis le dernier homme?

Je vais à Ferron comme à un père qui parle comme une mère. Ferron l'ambigu, l'inquiétant. II n'y a fondation que par oeuvre ondoyante. Ce qui fonde une langue, un peuple, un pays, ce ne peut être que la vie qui avance. Pour qui parle il n'y a de véritable fondation que fantastique. Ferron l'exemplaire: le bouc qui danse dans l'île verte de notre langue maternelle. Ferron l'interprète: celui qui sait la terre du cultiva teur et la ville de l'ouvrier, leur pauvretémisère et leur pauvreté-joie. 
Le Ciel de Québec, panoplie de héros québécois, d'héroïne québécoises, accomplit cette merveille de trouver la saveur de chaque individu peu importe son rang ou sa fonction dans la collectivité; quand la plume de Ferron touche un homme ou une femme il en sort la plupart du temps un héros ou une héroïne qu'il nous donne à rêver, à vivre, à faire nôtre. Cela est bon, d'autant plus que leur héroïsme n'est pas autrè chose qu' un entêtement à vivre leur vie, ce pourquoi ils et elles n'ont pas d'autre mérite que de suivre leurs sentier (quand l'espéce humaine marchait, les héros étaient des. dieux volants, maintenant que l'espèce piétine l'héroïsme tient à la marche: voyez comme on marche dans le Ciel de Québec, comme on se déplace).

1. Le texte est paru dans le no 90-91 de la Nouvelle Barre du Jour.

2. Adorno, cité par Henri Meschonnic dans Critique du rythme (1982). 